\title{
Design of the observation queue scheduler for WEAVE on the WHT
}

Fariña, Cecilia, Domínguez-Palmero, Lilian, Benn, Chris, Guest, Steve, Morris, Huw, et al.

Cecilia Fariña, Lilian Domínguez-Palmero, Chris Benn, Steve Guest, Huw Morris, Sergio Picó, Don Carlos Abrams, J. Alfonso L. Aguerri, Piercarlo Bonifacio, Esperanza Carrasco, Gavin Dalton, Kevin Middleton, Scott Trager, Antonella Vallenari, "Design of the observation queue scheduler for WEAVE on the WHT," Proc. SPIE 10704, Observatory Operations: Strategies, Processes, and Systems VII, 107040W (10 July 2018); doi: $10.1117 / 12.2312538$

SPIE Event: SPIE Astronomical Telescopes + Instrumentation, 2018, Austin, Texas, United States 


\title{
Design of the Observation Queue Scheduler for WEAVE on the WHT
}

Cecilia Fariña ${ }^{\mathrm{a}}$, Lilian Domínguez-Palmero ${ }^{\mathrm{a}}$, Chris Benn $^{\mathrm{a}}$, Steve Guest ${ }^{\mathrm{b}}$, Huw Morris ${ }^{\mathrm{b}}$, Sergio Picóa $^{a}$, Don Carlos Abrams ${ }^{\mathrm{a}}$, J. Alfonso L. Aguerric ${ }^{\mathrm{c}}$, Piercarlo Bonifacio ${ }^{\mathrm{d}}$, Esperanza Carrasco ${ }^{\mathrm{e}}$, Gavin Dalton ${ }^{\mathrm{f}, \mathrm{b}}$, Kevin Middleton ${ }^{\mathrm{b}}$, Scott Trager $^{\mathrm{h}}$, and Antonella Vallenari ${ }^{\mathrm{i}}$

${ }^{a}$ Isaac Newton Group of Telescopes, 38700 Santa Cruz de La Palma, La Palma, Spain

${ }^{b}$ RALSpace, STFC Rutherford Appleton Lab, Harwell Oxford, OX11 0QX, United Kingdom

'Instituto de Astrofísica de Canarias, 38200 La Laguna, Tenerife, Spain

${ }^{d}$ GEPI, Observatoire de Paris, Univ. PSL, CNRS, Place Jules Janssen, 92195 Meudon, France

${ }^{\mathrm{e} I N A O E}$, Luis Enrique Erro 1, Tonantzintla, Puebla, México

${ }^{\mathrm{f}}$ Dept. of Physics, University of Oxford, Keble Road, Oxford, OX1 3RH, United Kingdom

${ }^{\text {h }}$ Kapteyn Institut, RUG, Postbus 800, NL-9700 AV Groningen, Netherlands

${ }^{\mathrm{i} O s s e r v a t o r i o ~ A s t r o n o m i c o ~ d i ~ P a d o v a, ~ I N A F, ~ V i c o l o ~ O s s e r v a t o r i o ~ 5, ~} 35122$ Padova, Italy

\begin{abstract}
The Observation Queue Scheduler (OQS) for WEAVE is described in this paper, with particular emphasis on the scheduling algorithm. WEAVE is the new 2-deg field of view multi-object ( 1000 multiplex) spectroscopy facility $(\mathrm{R} \sim 5000$ and $\mathrm{R} \sim 20000)$ at the $4.2 \mathrm{-m}$ William Herschel Telescope. The OQS helps to maximize the scientific impact of WEAVE observations by optimising the schedule of the observing blocks, taking into account the science priority, required instrument configuration and observing constraints. On a nightly basis the OQS will assist the observer in creating a flexible queue of suitable observing blocks to be executed. It will be also possible to run a version of the OQS for extended periods of time to provide guidance on the longer-term planning of WEAVE surveys.
\end{abstract}

Keywords: WEAVE, observation queue scheduler, observatory control system, WEAVE operations, observing blocks, scoring model, scoring functions, scheduling algorithm

\section{INTRODUCTION}

A wide-field 1000-multiplex fibre-fed multi-object spectrograph, WEAVE, will be commissioned at the prime focus of the 4.2-m William Herschel Telescope (WHT) on La Palma by the end of 2019. Over a period of 5-10 years, WEAVE will carry out, in parallel, several surveys addressing a broad range of scientific themes*.

The unit of observation will be the observing block (OB). Each OB will contain all the information required to carry out simultaneous observation of up to $\sim 1000$ objects within the 2 -deg field of view. For maximum efficiency, a given OB will sometimes include targets from more than one of the science surveys. Each OB will define the instrument configuration and observing conditions required, and will have a given scientific priority. Each OB is for a total exposure time of approximately 1 hour.

Use of the observing night will be optimised with the help of an Observation Queue Scheduler (OQS), a software tool which incorporates two main components: (1) the scheduling algorithm which will build a queue of OBs to be observed during the night, taking into account the OB science priority, the instrument configuration and the required and predicted observing conditions and (2) the graphical user interface throught which the observer will be able to interact with the OQS during the night, e.g. to re-run it when observing conditions

Further author information: (Send correspondence to Cecilia Fariña)

E-mail: cf@ing.iac.es

*A description of WEAVE science cases can be found at: https://ingconfluence.ing.iac.es:8444/confluence/ /display/WEAV/Science.

Observatory Operations: Strategies, Processes, and Systems VII, edited by Alison B. Peck,

Robert L. Seaman, Chris R. Benn, Proc. of SPIE Vol. 10704, 107040W · @ 2018 SPIE

CCC code: $0277-786 \mathrm{X} / 18 / \$ 18 \cdot$ doi: $10.1117 / 12.2312538$

Proc. of SPIE Vol. 10704 107040W-1 
change, or when implementing tests or urgent override observations. All observations will be carried out in service mode by trained Isaac Newton Group staff observers.

This paper is organised as follows: WEAVE is introduced in Section 2, the requirements for the OQS are outlined in Section3, and the algorithm is described in Section 4. Section 5 gives examples of how special scheduling constraints will be handled. Section 6 describes the graphical user interface to the OQS. Section 7 summarizes the current status of the OQS, and the planned developments. Additional technical information is given in the appendices. Appendix A gives an overview of the software implementation, and Appendix B illustrates how moonlit sky brightness varies with the lunar phase, lunar elevation above the horizon and lunar angular distance.

\section{THE WEAVE MULTI-OBJECT SPECTROGRAPH AT THE WHT}

Below are described three aspects of WEAVE which are crucial for the design of the OQS: the properties of the instrument itself (Sect. 2.1), the Observing Block (Sect. 2.2), and the WEAVE operational model (Sect. 2.3).

\subsection{The WEAVE instrument}

The main components of the new instrument WEAVE are:

$\checkmark$ a new corrector for the WHT's prime focus, delivering a 2-deg field of view. ${ }^{1,2}$

$\checkmark$ a tumbler which can rotate between 0 and $180 \mathrm{deg}$ to position one of two plates, A and B, in the focal plane

$\checkmark$ a pick-and-place fibre positioner, which configures MOS fibres or integral-field units (IFUs) on one plate while observations are carried out with the other plate in the focal plane ${ }^{3}$

$\checkmark$ a two-arm (blue and red) fibre-fed spectrograph housed on one of the WHT's Nasmyth platforms ${ }^{4}$

$\checkmark$ a calibration unit which hosts a set of lamps for calibration images (arcs and flats)

The 3 focal-plane modes are:

$\checkmark$ MOS: Multi-object spectroscopy, provided by 960 and 940 configurable fibres on plate A and plate B, respectively. Each fibre subtends 1.3 arcsec on-sky. It's expected that most WEAVE observations will be carried out in MOS mode

$\checkmark$ mIFU: 20 mini-IFUs (each $11 \times 12 \operatorname{arcsec}^{2}$ ) on plate B

$\checkmark$ LIFU: Large IFU $\left(78 \times 90 \operatorname{arcsec}^{2}\right)$, positioned in the focal plane by rotating the tumbler to a position $90 \mathrm{deg}$ between those of plate A and plate B

The MOS-A, MOS-B, mIFU and LIFU modes use separate sets of $\sim 1000$ fibres (i.e. $\sim 4000$ in all) with each set terminating in a pseudo-slit module which can be moved into place at the focus of the spectrograph.

The spectrograph can be configured to deliver spectroscopic resolution of either $\mathrm{R} \sim 5000$ or $\mathrm{R} \sim 20000$.

At $\mathrm{R} \sim 5000$, the wavelength coverage is $\sim 366-465 \mathrm{~nm}$ in the blue arm and $579-959 \mathrm{~nm}$ in the red arm. At $\mathrm{R} \sim 20000$, the coverage is $\sim 404-465 \mathrm{~nm}$ or $473-545 \mathrm{~nm}$ (configurable) in the blue, and $595-685 \mathrm{~nm}$ in the red.

From the point of view of the OQS, then, the instrument can be used in several focal-plane modes and several spectroscopic modes. All changes to the configuration of the telescope, prime focus (including corrector, rotator and fibres), spectrograph and detector are carried out under computer control, i.e. can ultimately be driven by the OQS.

Other relevant configurable mechanisms within WEAVE include: the 3.5-arcsec guide-star fibre bundles (8 can be positioned on each plate), a 2-arcmin imager which can intercept light anywhere in the focal plane, and sources of calibration illumination positioned on the telescope (arcs and fibre flats) ${ }^{5}$ and within the spectrograph enclosure (for detector flats).

Additional information about WEAVE can be found in references 6 and 7. 


\subsection{The observing block}

The quantum of observation for WEAVE is the OB. Most OBs will take 1 hour to observe. This duration is set by several factors, the most important being the time (1 hour) it takes for the fibre-positioning robots to position $\sim 1000$ fibres on one plate. This limiting duration also keeps to an acceptable level the effects of differential refraction during observing (change of airmass effectively changes the scale in one direction, resulting in some targets not being exactly centred on the fibres). In addition, restriction of the observing time to 1 hour makes it more likely that the observing conditions are similar at the start and end of the OB.

For the WEAVE science surveys the OBs are defined by the WEAVE science teams. For WEAVE open time (part of the $30 \%$ of the telescope time not used for the WEAVE science surveys) the OBs are defined by the principal investigators.

Each OB is self-contained and defines the science observations and the configuration-dependent calibrations (e.g. fibre flats and arcs) necessary to reduce the data. A typical OB will define the following image set:

3 fibre flats -1 arc exposure $-3 \times(17$ minutes $)$ science observations -1 arc exposure

Each OB will also contain:

$\checkmark$ The field definition: RA and Dec of the field centre, fibre positions for science targets, guide stars and sky fibres, and relevant information about each target (e.g. proper motion, object type, magnitudes)

$\checkmark$ The instrumental configuration: observing mode (MOS - plate A/B, mIFU, LIFU) and diffraction gratings to be deployed into the light path in the spectrograph (defining the wavelength range and spectroscopic resolution)

$\checkmark$ The science priority

$\checkmark$ Required observing conditions (at the time of the OB being scheduled, and at that position on the sky):

- Maximum seeing (e.g. $<0.9$ arcsec)

- Brightest sky (e.g. $<21.5$ mag $\operatorname{arcsec}^{-2}$ )

- Minimum sky transparency (e.g. $>0.7$ )

- Minimum elevation (e.g. $>40 \mathrm{deg}$ )

- Minimum angular lunar distance (e.g. > $30 \mathrm{deg}$, because observations are particularly sensitive to any gradient in sky brightness across the field of view)

- Earliest and latest hour angle (e.g. because specific observations are particularly sensitive to wavelengthdependent loss of throughput caused by differential refraction)

- Additional constraints on the scheduling are envisaged for a small fraction of OBs (see Section 5.2)

The OBs are stored in an OB database, which can be interrogated by the observer at the telescope (see Appendix A).

\subsection{The WEAVE operational model}

Once commissioned, WEAVE will probably be on the telescope for at least $70 \%$ of nights, and will be used all night when observing conditions permit.

Observations at the WHT are currently (pre-WEAVE) carried out in visitor mode, with time being assigned by national time-allocation committees. Visiting observers have considerable freedom to adjust their observing programme during the night.

For a survey instrument such as WEAVE, a different approach is required, with an emphasis on long-term efficiency (reduced overheads, better quality control). Hence the queue-scheduling model, in which observations are planned well in advance, scheduled so as to make the best use of observing conditions, and carried out by trained staff observers rather than visitors. The staff observers will be able to make on-the-fly decisions which may override the OB queue proposed by the scheduling algorithm. Such decisions will usually be based on observing protocols agreed with the WEAVE science teams.

A summary of the WEAVE operational model is given below. 


\subsubsection{Preparation of OBs}

Well in advance of observing, and using dedicated software to calculate the positions of the fibres, ${ }^{8}$ the science teams will prepare the XML files containing the information required to create OBs. For MOS-mode observations, the number of fibres which can be positioned on science targets will depend on a number of factors, including the areal density of targets and how this varies across the field. $5-10 \%$ of the fibres will be used to sample sky (avoiding the positions of known stars). The science teams will also be responsible for selecting up to 8 guide stars well distributed across the field so as to provide both x,y and rotational tracking. For simplicity, each MOS field will be configured for only one of plate A or plate B. Fields are configured for observation on the meridian (hour angle $=0$ ). The XML files will be delivered to the observatory in batches of a few thousands, every few months. At the observatory, the XML files will be converted to actual OBs and will be ingested into an OB database.

The science teams will have access to an exposure-time calculator to check that the requested exposures will generate the signal-to-noise they require.

\subsubsection{Daytime activities at the telescope}

During the day, and during the part of twilight not needed for science observations, quality-control tests will be carried out, and some calibration observations will be taken. The illumination for the calibration observations will be provided by twilight sky or by lamps mounted on the cube of the telescope (to illuminate the focal plane with arc or continuum light) or inside the spectrograph (to illuminate the detectors directly). Most of the calibration observations will be obtained via OBs created on-the-fly, or copied from existing templates. Twilight observations will generally be taken at dusk rather than dawn.

The scheduling algorithm will also be run during the day, to generate a queue of 1-hour OBs to observe that night, taking into account the information given in the OB (especially RA and required observing conditions) and the current/predicted observing conditions. The first two MOS fields for the night will then be configured on plates A and B (to avoid wasting any configuration time at night).

At the start of the night, the observer checks the weather conditions, and may need to re-run the scheduling algorithm to take into account any changes in expected seeing or transparency.

\subsubsection{Observing}

Once start-of-the-night tests (e.g. telescope pointing) are completed, the observer instructs the OQS to execute the planned queue of observations. For a typical MOS observation, at any time during the night this requires (1) positioning of the fibres, (2) configuration of the instrument and telescope pointing, (3) acquisition of the field and closing the guide loop, (4) exposure and recording the resulting images, (5) quick-look data reduction.

Most of these actions are carried out automatically, and the observer is responsible for quality control, monitoring the weather, and handling interrupts.

Quality control includes ensuring that the field is correctly acquired and autoguided, via visual inspection of the real-time display of the autoguider images, and via a basket of metrics provided by the autoguider software. If a field cannot (after suitable on-sky testing) be acquired, the observation is cancelled (and the OB is flagged for inspection the next day) and it may be necessary to re-run the scheduling algorithm. The observer is also responsible for checking the results from the quick-look data reduction at the end of each exposure. In the event of the signal-to-noise being compromised due to weather or technical problems, the observer can elect to add an extra 20-min exposure.

The weather is monitored by a variety of devices. Near-zenith seeing is measured outside the WHT and elsewhere on site, and can also be monitored (in the direction of current WHT pointing) via the autoguider. Sky brightness is measured by a zenith-pointing camera outside the WHT, and a similar camera is mounted on the WHT top-end ring. Transparency is monitored via feedback from the autoguider (the guide stars are of known magnitude).

Interrupts can arise as a consequence of technical problems, bad weather or science overrides. Protocols will be in place to deal with each kind of interrupt, and many of these will involve re-running the scheduling algorithm for the rest of the night. 


\section{THE OBSERVING QUEUE SCHEDULER SYSTEM REQUIREMENTS}

The overall requirements for the OQS are that it helps optimise the scientific use of the night, through appropriate queuing of the OBs, and that it be responsive and straightforward to use. Optimal use of the night implies that individual OBs are observed under the required conditions (seeing, sky brightness, etc.), that observing overheads are minimised, and that scientific priorities are respected (e.g. that no unnecessary biases result in some surveys getting more on-sky time than intended).

More formally, the requirements for the OQS software are as follows:

$\checkmark$ It must create an optimised queue of OBs, taking into account the OB-specified science priorities, instrument configuration (and any current technical constraints on this), position of the field on the sky, observing conditions required and the estimated observing conditions at the time the OB is queued (and at the location on the sky at which the OB is queued).

$\checkmark$ It must allow the queue of OBs to be re-optimsed at any time, including during the night, e.g. when observing conditions change.

$\checkmark$ It must allow creation of a long-term (many nights) queue, to facilitate planning.

$\checkmark$ It must allow modification of the queue on the fly, to add or remove OBs, or adjust the order of observing.

$\checkmark$ It must be possible to modify (1) the numerical parameters defining the scheduling algorithm and (2) any scheduling constraints (e.g. if a particular observing mode becomes unavailable because of a technical problem).

The OQS is a decision-management system and as such, incorporates two key components: the scheduling algorithm, and the graphical user interface.

The scheduling algorithm (Section 4): This takes as input the list of OBs to be observed, and the associated constraints, and delivers as output a queue of OBs for the night (or nights) in question. For each time slot in the queue, the OQS also lists all the other OBs which were most-highly ranked for observation at that time.

The graphical user interface (Section6): This is a tool for visualisation and for interaction with the scheduling algorithm during planning for a given night, and also during the night itself. It will be used to generate and modify the queue, adjust the algorithm parameters, execute individual OBs and visualise the queue in various ways.

The scheduling algorithm itself can be run from a command line with options passed via a series of arguments. This is being used extensively for testing purposes, and will also be useful for longer-term survey planning, when it can be run using simulated or historical observing conditions.

\section{THE OBSERVATION QUEUE SCHEDULING ALGORITHM}

The previous sections depict the main requirements and functionalities that drive the design for the observation queue scheduler. In summary, WEAVE is a single instrument with three possible observing modes. The observing modes are independent for the instrument but they are not independent for the scheduler because of the 1-hour MOS configuration time and the need to minimize overheads. The MOS configuration time is a constraint imposed by the WEAVE instrument design which affects the WEAVE operations and therefore the scheduler: Most of the OBs that will feed the scheduler will take 1 hour to observe (including fibre dependent calibrations) and the scheduler has to evaluate the OBs observing constraints at least one hour and a half in advance of the observation of the OB. A significant change in the observing conditions after starting the configuration may require the scheduler to be run again, potentially incurring overheads if the queue is modified. Certain conditions as seeing, can vary (in quite unpredictable way) in a short time scale during the night having an impact on the signal to noise of the observations. Therefore, the scheduler algorithm has to be agile, efficient, and flexible. Agile to run in real time during the observing night as the observing conditions change. Efficient to always choose an optimized set of OBs to observe according to the new estimated conditions. Flexible to allow modifications, as the situation requires, both in the parameters used by the algorithm to make the queue and also in the resulting queue itself as there will be always an observer to make the final decision on the OBs scheduled for observation. 
The conditions described above motivate the implementation of the dispatch scheduler model, extensively analysed by Fraser $(2012)^{9}$. The WEAVE scheduling algorithm works as follows: it scans the OB database and selects the OBs that, given their Right Ascensions, might be observable. From this subset of observable OB the scheduling algorithm makes a ranking of the best candidates for observation, by matching the current/estimated observing conditions with the OBs observing constraints and priorities. The ranking is made by assigning a score to each OB.

\subsection{Scoring model, scoring functions, weights, and constraints}

The scoring model used by the algorithm comprises a set of well-defined scoring functions $\left(f_{i}\right)$ and their respective weights $\left(w_{i}\right)$. The OB total score, the number used to rank each OB for each time slot is:

$$
\begin{array}{rlrl}
S_{\text {total }}=\sum_{i=1}^{n} w_{i} f_{i}(t) & \text { if all } & f_{i}(t) \neq 0 \\
S_{\text {total }}=0 & \text { if any } & & f_{i}(t)=0
\end{array}
$$

where the index ' $i$ ' represents a parameter relevant to observation of the OBs (seeing, sky transparency, sky brightness, elevation, etc.).

Time slot in this context means the time it takes to observe an OB from beginning to end. The scoring functions are evaluated at a time t within this 1-hour time-slot, usually at the mid-point of the slot, but sometimes at the beginning and/or the end.

A scoring function, $f_{i}(t)$, is defined for each of the relevant parameters $\mathrm{i}$, at time $\mathrm{t}$. The OBs limit the allowed ranges of values of some of these parameters. The allowed values of some parameters will also be constrained by instrument, telescope and operational issues. If any scoring function $f_{i}=0$, the OB is not considered for time t.

A weight, $w_{i}$, is associated with each scoring function. The weights can be used to: (1) Tweak the relative importance given to one constraint relative to the others and (2) eliminate individual parameters from the total score by setting $w_{i}=0$. During most observing, the algorithm will use a default set of weights. However, the weights can be modified by the observer as and when required.

Some parameters define hard constraints. Others define optimisable constraints:

Hard constraints: Each of these constraints must be met for the OB to be observable. The scoring function for each hard constraint therefore has value 0 or 1. $f_{i}(t)=0$ produces $S_{\text {total }}=0$ for the OB, so the OB will not be considered for the time slot ' $t$ '. These functions act as a filter for the OBs.

Some of the hard constraints are:

$\checkmark$ Telescope constraints (minimum WHT elevation, WHT blind spot, etc.) $\rightarrow$ specified by the observatory

$\checkmark$ Instrument constraints (e.g. due to temporary technical failures) $\rightarrow$ specified by the observatory

$\checkmark$ Minimum elevation $\rightarrow$ specified in the OB by science teams

$\checkmark$ Minimum angular distance of the moon $\rightarrow$ specified in the OB by science teams

$\checkmark$ Observing mode availability $\rightarrow$ depends on the observing mode used in the previous scheduled OB

$\checkmark$ Observing-conditions limits specified by the observatory to avoid observing in conditions which might have dire but non-obvious implications e.g. loss of autoguiding if the seeing is very bad, light scattered off telescope structure if moon too close, increased risk of rain as transparency drops. Current values set in the scoring functions described in Sect. 4.2 are: brightest sky $V>17 \mathrm{mag} \mathrm{arcsec}^{2}$ (twilight), seeing $<3 \operatorname{arcsec}$, sky transparency $>0.4$, angular distance from the moon $>20 \mathrm{deg}$, and elevation $>20 \mathrm{deg}$.

Optimisable constraints: The scoring function for each of the optimisable constraints indicates how well the OB matches the observing opportunity at time 't' given e.g. the hour angle and the prevailing and required observing conditions. Again $f_{i}(t)=0$ results in $S_{\text {total }}=0$, i.e. the OB is not observable in the time slot. 
Optimisable constraints include:

$\checkmark$ Observing-conditions constraints: seeing, sky transparency, sky brightness, hour-angle limits $\rightarrow$ specified in the OB by science teams

$\checkmark$ Observing priorities: science priority, Intra-survey priority, override priority (see Sect. 4.2.5)

$\checkmark$ Overheads: telescope slew, instrument setup, fibre configuration $\rightarrow$ specified by the observatory

\subsection{Scoring functions for optimisable situations}

The aim of the scoring functions for optimisable constraints, is to rank higher those OBs for which the current observing conditions best match the conditions requested in the OBs, while at the same time taking into account the probability with which different observing conditions are encountered.

The scoring functions were designed to mimic the choice that a human scheduler would do, that is: (1) select to observe those OBs with more restrictive conditions (hence, less probable to occur) when they are met and (2) observe each individual $\mathrm{OB}$ in its most favorable situation. To illustrate this it helps to think about a specific example e.g. the scoring function for the seeing. Lets OB1 and OB2 have maximum seeing constraint of 0.9 and 1.5 arcsec, respectively and the estimated seeing for the time slot be 0.7 arcsec. In such situation the scoring function will be higher for OB1 than for OB2 (as OB1 requires more restrictive seeing, that happens less often). On the other side, for different estimated seeing conditions the seeing scoring function will favor to observe an $\mathrm{OB}$ in the lowest seeing available. Therefore, the scoring functions are 2-dimentional surfaces where each OB idelally fill most of the range between a minimum of 0 (OB cannot be scheduled at this time slot) and a maximum of 1 .

All the scoring functions are dimensionless and normalised to fall within the range 0 - 1 . Constant values in the extremes of the parameters (as maximun and minimum possible seeing values during a night) were used to define the functions in a physically meaningful range. Table 1 lists the six optimisable scoring functions and relevant information. The characteristics of the scoring functions are described in detailed in the following sections where each scoring function is presented.

Table 1. Optimisable scoring functions and the associated parameters.

\begin{tabular}{|c|c|c|c|c|}
\hline Parameter & Scoring Function & Constraints in the $\mathrm{OB}$ & Fixed parameter values & Section \\
\hline Seeing & $f_{\text {seeing }}$ & $\begin{array}{l}\text { Maximum seeing: } \\
\text { seeing } \\
\text { B } \max \end{array}$ & $\begin{array}{c}\text { seeing_Max }=3 \\
\text { seeing_Min }=0.3\end{array}$ & 4.2 .1 \\
\hline $\begin{array}{c}\text { Sky } \\
\text { Brightness } \\
\end{array}$ & $f_{s b}$ & $\begin{array}{c}\text { Brightest sky: } \\
\text { sboBbright }\end{array}$ & $s b \_M i n=22$ & 4.2 .2 \\
\hline $\begin{array}{c}\text { Sky } \\
\text { Transparency }\end{array}$ & $f_{\text {stra }}$ & $\begin{array}{c}\text { Minimum sky transparency: } \\
\text { stra }_{O B \min }\end{array}$ & stra_Max $=0.88$ & 4.2 .3 \\
\hline $\begin{array}{l}\text { Hour Angle } \\
\text { range }\end{array}$ & $f_{H A}$ & $\begin{array}{l}\text { Earliest and latest hour angles: } \\
\qquad H A_{O B \min }, H A_{O B \max }\end{array}$ & - & 4.2 .4 \\
\hline Priority & $f_{O B t p}$ & $\begin{array}{c}\text { Science priority: } P_{O B s c i} \\
\text { Override priority: } P_{O B t o v r}\end{array}$ & - & 4.2 .5 \\
\hline Overheads & $f_{\text {overheads }}$ & - & - & 4.2 .6 \\
\hline
\end{tabular}

\subsubsection{Scoring function for seeing: $f_{\text {seeing }}$}

Seeing varies approximately as airmass ${ }^{0.6}$ :

$$
\left.\operatorname{seeing}(\text { airmass }, t)=\operatorname{airmass}_{O B}(t)^{0.6} \times \operatorname{seeing}_{(\text {zenith }, t}\right)
$$


Hence, if seeing $=1.0 \operatorname{arcsec}$ at the zenith it will be $\sim 1.3 \operatorname{arcsec}$ at airmass $=1.5$, and $\sim 1.5 \operatorname{arcsec}$ at airmass $=2.0$.

For seeing $($ airmass, $t) \leq$ seeingoBmax

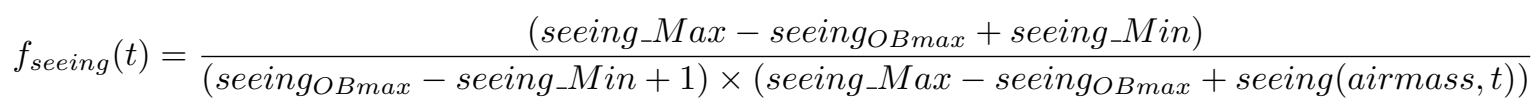

For seeing $($ airmass, $t)>$ seeingoBmax

$$
f_{\text {seeing }}=0
$$

where all the seeing values in Eqn. 3 are in units of arcsec, and:

- seeing_Max and seeing_Min are configurable values for the maximum and minimum possible seeing values during a night. Current values in use are seeing_Max $=3$ and seeing_Min $=0.3$

- seeing $_{O B \max }=$ Maximum OB seeing

- seeing (airmass, $t)$ is the predicted seeing for the time ' $t$ ' for the OB airmass by Eqn. 2
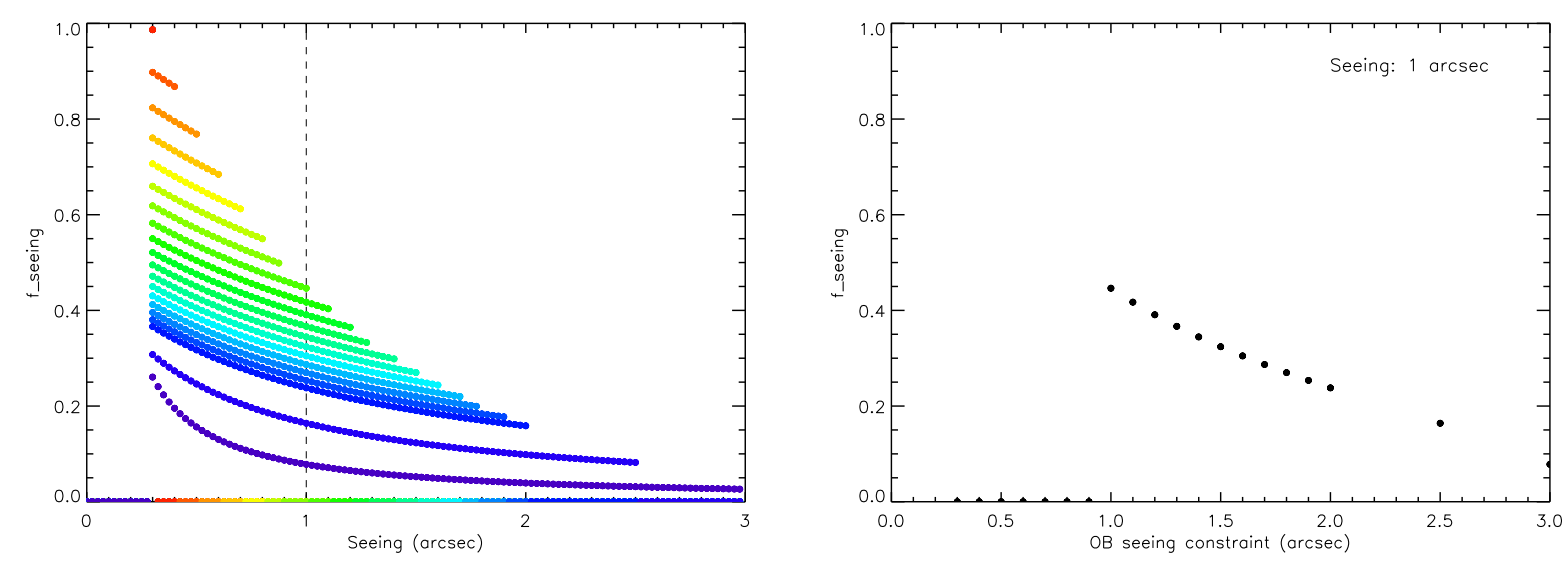

Figure 1. Left panel: $f_{\text {seeing }}$ as a function of the current seeing from Eqn. 3, for each of several values of OB-specified maximum, seeing $g_{O B \max }$, from seeing $g_{O B \max }=0.3$ (red) to 3 arcsec (violet). Right panel: $f_{\text {seeing }}$ as a function of OBspecified maximum seeing, seeing $g_{O B \max }$, from Eqn. 3 for a fixed seeing value seeing airmass $=1$ arcsec, i.e. the $f_{\text {seeing }}$ values intercepted by the dashed line in the left hand plot.

\subsubsection{Scoring function for sky brightness: $f_{s b}$}

The scheduling algorithm calculates the sky brightness at the position of each OB, taking into account the expected contributions from scattered moonlight, airglow and zodiacal light (see Appendix B for further discussion). During operation of WEAVE, the algorithm may also take into account real-time measurements from the two sky-brightness monitors mentioned in Sect. 2.3.3.

Note about the variation of sky brightness:

$\checkmark$ The dark-of-moon sky will be brighter (than at zenith) by a factor $\sim 1.3$ at airmass $=1.5$, and $\sim 1.6$ at airmass $=2.0$. These factors are for solar minimum (next minimum is expected $\sim 2020$ ) but vary only a little with solar cycle.

$\checkmark$ The dark-of-moon sky is brighter by a factor $\sim 1.4$ at low ecliptic latitude compared to high ecliptic latitude. The variation with galactic latitude is small. 
$\checkmark$ When the moon is up, scattered moonlight is the main contribution to sky brightness. Moonlit sky brightness depends strongly on the phase of the moon and angular distance from the moon and, less strongly, on the elevation of the moon (see appendix B for more details). E.g. at full moon, for typical source and moon elevations, for target-moon separations of 10,40, and $80 \mathrm{deg}$, the sky is brighter than dark-of-moon by $\sim 4.1,3.2$, and 2.6 mag $\operatorname{arcsec}^{-2}$, respectively.

$\checkmark$ As described in Sect. 2.2, each OB sets a minimum angular distance from the moon and in addition, the scheduling algorithm sets a minimum moon distance to minimise the risk of scattered moonlight reaching the focal plane. Both limits are treated as hard constraints. The current value for the telescope moon distance limit is $20 \mathrm{deg}$. This value may change after commissioning.

For $s b_{O B}(t) \geq s b_{O B b r i g h t}$

$$
f_{s b}(t)=\frac{\left(s b_{O B b r i g h t}+1\right)}{\left(s b_{-} \text {Min }-s b_{O B b r i g h t}+1\right) \times\left(s b_{-} \text {Min }-s b_{O B}(t)+s b_{O B b r i g h t}+1\right)}
$$

For $s b_{O B}(t)<s b_{O B \max }$

$$
f_{s b}=0
$$

where all the sky brightness values in Eqn. 4 are in units of $\operatorname{mag} \operatorname{arcsec}^{-2}$, and:

- $s b$ Min is a configurable value for the darkest possible sky brightness value during a night in V-band magnitudes. Currently used value is $s b \_$Min $=22$

$\cdot s b_{O B b r i g h t}=$ Brightest OB sky

- $s b_{O B}(t)$ is the predicted sky brightness for the time ' $t$ ' for each OB according to its position, moon distance, moon phase, etc. (see plots in Appendix B for reference)
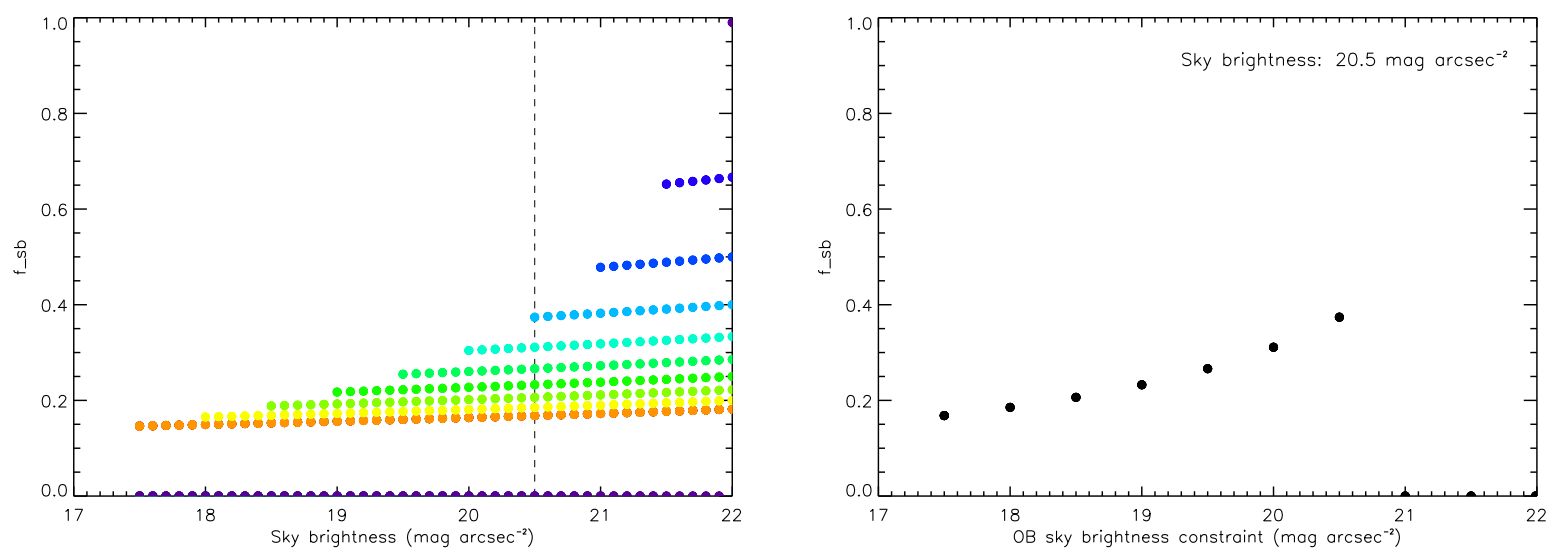

Figure 2. Left panel: $f_{s b}$ as a function of the expected sky brightness from Eqn. 4, for each of several values of OB-specified brightest acceptable sky, $s b_{O B b r i g h t}$, from $s b_{O B b r i g h t}=22$ (violet) to $17.5 \mathrm{mag} \operatorname{arcsec}^{-2}$ (orange). Right panel: $f_{s b}$ as a function of OB-specified brightest acceptable sky, $s b_{O B b r i g h t}$, from Eqn. 4 for a fixed sky brightness value $s b_{O B}=20.5$ mag $\operatorname{arcsec}^{-2}$, i.e. the $f_{s b}$ values intercepted by the dashed line in the left hand plot. 


\subsubsection{Scoring function for sky transparency : $f_{\text {stra }}$}

On most cloudless, non-dusty nights, the V-band transparency at the zenith (i.e. the fraction of light transmmitted by the atmosphere), is $\approx 0.88$ and it probably scales approximately as $0.88^{\text {airmass }}$. If at zenith sky transparency is stra $_{\text {zenith }}=0.88$, it will be $\sim 0.83$ at airmass $=1.5$, and $\sim 0.77$ at airmass $=2$.

The scheduling algorithm does not take into account this variation of sky transparency with airmass because the dominant effect on sky transparency, at the elevation limits of the majority of OBs, is cloud (usually not homogeneous) and, during a few nights a year, Saharan dust. The scheduling algorithm will use a unique sky transparency value for all the OBs independently of the OB position in the sky. The scheduler will fetch the values of the sky transparency at telescope position from the Autoguider, it will average previous measured values for sky transparency and use that value as $\operatorname{stra}(t)$ to evaluate the $f_{\text {stra }}(t)$ function for all the OBs in each time slot.

For $\operatorname{stra}(t) \geq \operatorname{stra} a_{O B \min }$

$$
f_{\text {stra }}(t)=\frac{\left(\text { stra }_{\text {OBmin }}+1\right)}{\left(s t r a \_M a x-s t r a_{O B m i n}+1\right) \times\left(\text { stra_Max }-s t(t)+s t r a_{O B m i n}+1\right)}
$$

For $\operatorname{stra}(t)<s t r a a_{O B i n}$

$$
f_{\text {stra }}(t)=0
$$

where all the sky transparency values in Eqn. 5 are dimensionless, and:

- stra_Max is a configurable value for the maximum possible sky transparency value during a night. Currently used value is stra_Max $=0.88$

- stra $_{O B \min }=$ Minimum OB sky transparency

- $\operatorname{stra}(t)$ is the predicted sky transparency for time 't'. For $0<\operatorname{stra}(t)<0.4$ the telescope will probably be closed
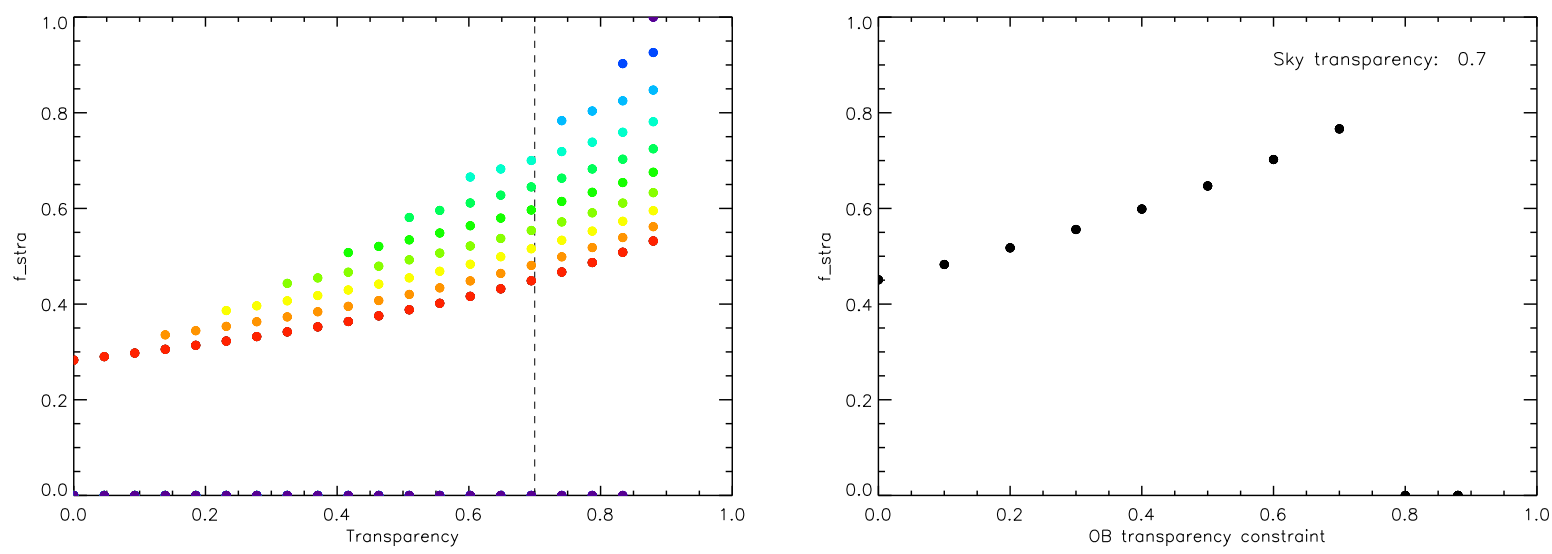

Figure 3. Left panel: $f_{\text {stra }}$ as a function of the expected sky transparency from Eqn. 5, for each of several values of OBspecified minimum sky transparency, stra $a_{O B \min }$, from $s t r a_{O B \min }=0.88$ (violet) to 0.0 (red). For values of $s t r a(t) \lesssim 0.4$ the observations will probably be interrupted. Right panel: $f_{\text {stra }}$ as a function of OB-specified minimum sky transparency, stra $a_{O B \text { min }}$, from Eqn. 5 for a fixed sky transparency value $\operatorname{str} a_{O B}=0.7$, i.e. the $f_{\text {stra }}$ values intercepted by the dashed line in the left hand plot. 


\subsubsection{Scoring function for hour angle: $f_{H A}$}

As described in Sect. 2.2, each OB inherits (from the configuration software) limits on the earliest and latest hour angles (HA) at which the configuration is valid. Configuration outside these limits could result in collisions between fibres because their positions on the field plate will shift to compensate for differential refraction. The fibre positions in the OB field plate definition are associated with a certain HA $\left(H A_{\text {conf }}\right)$ which is usually close to the field culmination $\left(H A_{\text {conf }} \sim 0\right)$. At the telescope the field will be configured for the HA that falls in the middle of the time slot assigned to the $\mathrm{OB}\left(H A\left(t_{c}\right)\right)$, to minimise the effect of differential refraction during observation. When $H A_{\text {conf }} \neq H A\left(t_{c}\right)$ the fibre positioner will correct the positions of the fibres to allow for differential refraction. Hence, if $H A\left(t_{c}\right)$ falls outside the hour-angle limits, the fibre offsets might cause some fibres to collide (although in practice, the robots will automatically park fibres to avoid the collision).

- $H A_{O B \max }=$ Latest possible HA for configuring the field (hours)

- $H A_{O B \min }=$ Earliest possible HA for configuring the field (hours)

- $H A_{H}=\mathrm{HA}$ at the highest elevation within range $_{O B}$.

- $H A_{O B}(t)=$ calculated HA of the OB at time ' $\mathrm{t}$ '

- $t_{c}$ time at the middle of the OB observation

- range $_{O B}=\left|H A_{O B \max }-H A_{O B \min }\right|$

- dist $=\left|H A_{O B}\left(t_{c}\right)-H A_{H}\right|$

There will be three regimes of HA values as follows:

1. If $H A_{O B \min } \leq 0$ and $H A_{O B \max } \geq 0 \rightarrow H A_{H}=0$

2. If $H A_{O B \min }<0$ and $H A_{O B \max }<0 \rightarrow H A_{H}=H A_{O B \max }$

3. If $H A_{O B \min }>0$ and $H A_{O B \max }>0 \rightarrow H A_{H}=H A_{O B \min }$

$$
f_{H A}(t)=\frac{1}{\left(\text { range }_{O B}+1\right) \times(\text { dist }+1)}
$$

For $H A_{O B}\left(t_{c}\right)<H A_{O B \min }$ and $H A_{O B}(t c)>H A_{O B \max }$

$$
f_{H A}=0
$$

All the HA values in Eqn. 6 are in units of hours.

\subsubsection{Scoring function for OB priority: $f_{O B t p}$}

The scheduling algorithm uses a set of priorities to calculate the total OB priority:

$\checkmark$ OB science priority $\left(P_{O B s c i}\right)$ : Each OB has a science priority assigned by the science teams (see explanation in Sect. 2.2).

$\checkmark$ Override priority $\left(P_{O B o v r}\right)$ : This is an internal priority from the scheduling algorithm. It will be used, for a limited time, on particular occasions, to bias the scheduling. This priority is to be used only on request of the science teams. $P_{O B o v r}=1$ for nearly all the OBs most of the time. 


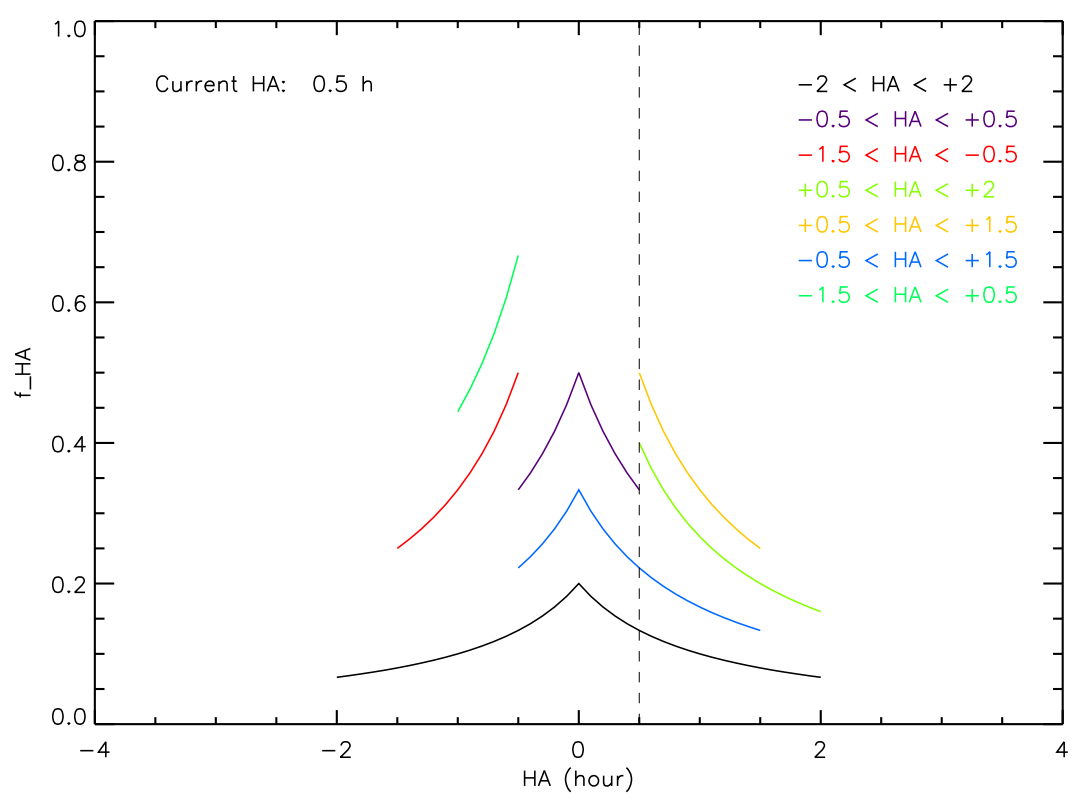

Figure 4. $f_{H A}$ as function of the OB Hour Angle $\left(H A_{O B}(t)\right)$ from Eqn. 6. Each color corresponds to the set of values of the earliest and latest $\mathrm{OB} H A$ constraints $\left(H A_{O B \min }\right.$ and $\left.H A_{O B \max }\right)$ indicated in the figure reference. $f_{H A}$ reaches its maximum for each $\mathrm{OB}$ at the closest $\mathrm{HA}$ to the $\mathrm{OB}$ culmination $\left(H A_{O B}=0\right)$, that is the most favorable situation in with to observe an OB. For OBs with larger range of HA $\left(\right.$ range $O B$ ), the function $f_{H A}$ gets lower values, as these OBs has a higher chance to be observed.

$$
f_{\text {OBtp }}=P_{\text {OBsci }} \times P_{\text {OBtovr }}
$$

with $0<P_{O B s c i} \leq 1$

There is also an Intra-survey priority $\left(P_{\text {OBint }}\right)$, which is an internal priority from the scheduling algorithm. This priority modifies the priority of an OB relative to the other OBs in the same survey. This is used to handle special scheduling requests explained in Sect. 5.2.

\subsubsection{Scoring function for OB overheads: $f_{\text {overheads }}$}

$f_{\text {overheads }}$ decreases as the overheads (telescope slew, instrument setup, fibre configuration) increase. Accurate overheads will be measured during the commissioning.

$$
f_{\text {overheads }}=1-0.9 \times \text { Overheads }
$$

\section{SPECIAL SCHEDULING SITUATIONS}

Special scheduling constraints arise at the beginning and end of the night (Sec.5.1) and as a result of unusual science requirements (Sec. 5.2).

\subsection{Beginning and end of the night}

Twilight at the beginning and end of the night can be used for observing those science OBs whose sky-brightness constraints allow it. This is particularly relevant for the moonlit part of a given night, when the sky-brightness of the scheduled OBs is necessarily less stringent, effectively extending the usable night into at least one twilight by $\sim 0.5$ hour (when twilit sky brightness approaches that due to moonlight). 
The scheduling algorithm is designed to make optimum use of the twilights. This time is ideal for making short observations required to complete OBs which have not had their full required exposure time. Such observations are usually expensive to make during the night because such a science observation completes in a shorter time than required to configure a MOS plate, effectively incurring extra overheads. But if the fibre configuration for the short observation is ready by the start of the night (i.e. is made in the afternoon), there are no extra overheads. Similarly, at the end of the night, after the last OB observed entirely during astronomical night, the algorithm will attempt to include an $\mathrm{OB}$ which fits in the remaining time (end of astronomical night plus twilight) without violating the sky-brightness constraint.

\subsection{Special scheduling constraints triggered by science}

WEAVE is being used mainly for large surveys, so the order in which the OBs are observed is usually not impotant, as long as the required observing conditions are met. However, for a small fraction of OBs, the order of observation relative to specified other OBs is important. In such cases, either the scheduling algorithm can be extended to cater for the requirement, or protocols can be put in place to handle the requirement through manual intervention.

The cases which have come to light so far are described below:

$\checkmark$ Fields requiring more than 1 hour of integration time (with no deadline):

The total integration time for these fields will be achieved by generating identical, 1-hour, chained OBs adding up to the total integration time. The scheduling requirement is that once a chain of OBs is started for a given field, this field should be prioritised (by the software) relative to other fields for the same science survey, and in the same time slot, in order to ensure, where possible, completion of the long integration before starting observation of another field in that science survey.

To achieve this, if one chained OB is selected, the system will increase the OB Intra-survey priority (Sec. 4.2.5) of the rest of OBs of this chain relative to the other OBs in the same survey. For each time slot the algorithm will rank the OBs and select an OB from all the possible surveys. Once this selection is made there will be another intra-survey competition for the time slot, as the scheduling algorithm will choose, from the ranked OBs, the OB (within the survey of the original selected $\mathrm{OB}$ ) with the highest $P_{\text {OBint }}$. Then this OB will be scheduled in the observing queue (overriding the original order given by the $\left.S_{\text {total }}\right)$. $P_{\text {OBint }}$ will be increased each time one of the chained OBs from the set is observed to favor completion of the whole set of chained OBs before starting a new one.

$\checkmark$ Field-specific science-driven scheduling constraints:

These fields will comprise linked 1-hour OBs, whose configurations are not necessarily identical (although they share most of the targets, they may be OBs defined on different field plates). The scheduling requests for these fields will be communicated by the science teams to the observatory. These requests will be handled through manual intervention by the observer. The linked-OBs will share a special code that allows identification of them as a set and with the particular scheduling request. This code will be displayed to the observer who will review the scheduling request and take the necessary actions to fulfull it.

\section{THE GRAPHICAL USER INTERFACE}

During the observing nights the observer will use the OQS via the GUI which

$\checkmark$ displays the observation queue (in different formats), its status, and a series of warnings when conditions are not met

$\checkmark$ displays the current status of the observations being executed

$\checkmark$ displays relevant information about the observing conditions

$\checkmark$ provides an interface through which the observer will be able to interact with the OQS to edit the queue and tweak the scheduling-algorithm weights.

$\checkmark$ allows interaction with the Sequencer to execute observations 
The GUI embodies three main tabs:

1. Observing tab

2. OB-database tab

3. Parameter-settings tab

\section{The Observing tab}

The observer will interact with the OQS mainly through the observing tab, which will be prominently displayed to him/her throughout most of the night. The panels on this tab are, following the numbering on Fig. 5:

1. General-information panel: displays information about the current observing conditions at zenith and at the current telescope position. It also displays the Local Sidereal Time (LST) and the Universal Time (UT)

2. OB queue panel: a display and interactive table that shows the current queue with relevant information (and warnings) about the OBs in the queue.

3. Action buttons panel: a set of buttons with actions to modify the queue and to command the Sequencer to execute an $\mathrm{OB}$ (configure and observe).

4. Queue visibility panel: displays the visibility plot (UT as function of elevation/airmass) for the OBs currently in the queue, highlighting the period of observation for each OB and its distance to the moon. The visibility of the moon is also shown.

5. All-sky panel: an all-sky image from a camera on-site, updated every few minutes, showing the positions of the objects in the queue.

The queue table, visibility plot and the all-sky panel are interactive: clicking on any OB in one of the panels highlights the same OB in the other two panels.

\section{The OB-database tab}

From this tab the observer can interact with the OB database, e.g. to include additional OBs in the queue. This table allows one to search on OBs using different criteria, select and send OBs to the current queue, etc.

\section{Parameter-settings tab}

From this tab the observer can interact with the OQS, e.g. to create or modify the queue, set the weights $w_{i}$ of the scoring functions, specify the observing conditions or change the telescope/instrument constraints.

\section{CURRENT STATUS AND PLANNED TESTS}

The OQS is well advanced, with most of the requirements implemented, allowing it to be used in a recent WEAVE 'operational rehearsal' exercise. For this particular exercise ('Operational Rehearsal 3'), the OB database was populated with 3 months worth of mock (but representative) OBs provided by the WEAVE science teams, and the scheduling algorithm was run to optimise the schedule for each night in each of the 3 months September 2016, February 2017 and September 2017, using the actual observing conditions recorded during those 3 months, and the actual lunar phase and position on the sky.

The results of the rehearsal are shown in Table 2. The scheduling algorithm performed well, with the main problem being a paucity of bright-time and poor-seeing OBs amongst the submitted OBs for Sep-2016 and Feb-2017. For the Sep-2017 schedule, some of the OB constraints on observing conditions were reviewed and this resulted in a higher fraction of the time being scheduled for observation. When WEAVE is on-sky, the over-subscription (column (4) of Table 2) will be much higher, but this exercise serves as a timely reminder that, averaged across all of the science surveys being carried out by WEAVE, the time requested for each combination of observing conditions needs to match that available. 




Figure 5. Snapshot of the 'observing tab' of the GUI, in its current state of development. Panel (1) in the uppermost part of the image gives information about the current observing conditions. The table in (2) displays the current queue for the night with relevant information about each OB. If any of the observing-conditions constraints are no longer fulfilled, a warning will appear in the relevant column of the entry for that OB. The buttons in panel (3) allow the observer to modify the queue and to execute the OBs. The visibility plot in (4) shows the elevation/airmass for each scheduled OB and for the moon. The image in (5) displays the current visible hemisphere of the sky with the positions of the scheduled OBs indicated.

The most complicated aspects of the OQS design are the scheduling algorithm and the graphical user interface. These are being designed iteratively, through close collaboration between observatory staff and software engineers, with thorough testing between iterations.

Testing the scheduling algorithm (and tweaking its coefficients) is particularly demanding, involving exploration of a very large parameter space.

Planned tests of the algorithm include the following:

$\checkmark$ Testing how well the scoring functions influence the scheduling algorithm as the observing conditions are varied.

$\checkmark$ Checking the reaction of the OQS after various types of observing interrupt, e.g. bad weather or technical problems. 
Table 2. Results of WEAVE operational-rehearsal exercise. Column 1 gives the scheduled month. Column 2 is the total number of hours available for each month (including bad weather and 0.5 hour extra per night for afternoon and morning twilights). Note that for Sep-2017 29 nights were considered and for Feb-2017 27 nights, due to limited availability of historical weather conditions in the database. Column 3 is the number of 1-hour OBs available. Column 4 is the oversubscription, i.e. the total available OBs (Column 3) divided by the total available time (Column 2). Column 5 lists the number of scheduled OBs. Column 6 is the percentage of time used for observing. Column 7 is the percentage of time lost due to bad weather (from the weather database). Column 8 is the percentage of time potentially usable but for which no OBs were scheduled mainly due to paucity of bright-time and poor-seeing OBs amongst the submitted OBs.

\begin{tabular}{|c|c|c|c|c|c|c|c|}
\hline Period & $\begin{array}{c}\text { Total } \\
\text { hours } \\
(2)\end{array}$ & $\begin{array}{c}\text { Totald } \\
\text { OBs } \\
(3)\end{array}$ & $\begin{array}{c}\text { Over- } \\
\text { subscription } \\
(4)\end{array}$ & $\begin{array}{c}\text { Schedule } \\
\text { OBs } \\
(5)\end{array}$ & $\begin{array}{c}\text { Observed } \\
\text { Time (\%) } \\
(6)\end{array}$ & $\begin{array}{c}\text { Time lost } \\
\text { Weather (\%) } \\
(7)\end{array}$ & $\begin{array}{c}\text { Time not } \\
\text { used (\%) } \\
(8)\end{array}$ \\
\hline Sep-2016 & 276 & 407 & 1.5 & 203 & 80 & 0 & 20 \\
\hline Feb-2017 & 288 & 396 & 1.4 & 143 & 54 & 26 & 20 \\
\hline Sep-2017 & 285 & 578 & 2.0 & 239 & 91 & 6 & 3 \\
\hline
\end{tabular}

$\checkmark$ Studying the effect of instrumental/telescope constraints (such as the blind-spot near the zenith) on the selection of OBs.

$\checkmark$ Varying the weights $w_{i}$ for the individual scoring functions, to allow standard (default) sets to be selected.

$\checkmark$ Expanding the simulation reported above, to cover a few years of observations, using historical observing conditions and drawing on a set of mock OBs which is realistic in terms of size, distribution on the sky and distribution of requested observing conditions.

On a broader front, the interactions between the OQS and the other software components of the observatory control system also need further testing. 


\section{APPENDIX A. SOFTWARE IMPLEMENTATION AND ENVIRONMENT}

The OQS is written in Python3.5 and uses the packages: PyEphem, numpy, astropy, matplotlib, PyGreSQL, postgresql, wxPython Phoenix, and pytest.

The OQS communicates directly with three subsystems of the OCS:

$\checkmark$ The Parameter Noticeboard: At regular intervals, the OQS fetches, from this, the latest information about the current observing conditions (e.g. seeing, sky brightness, transparency), the fibre positioner and the Sequencer information (see below).

$\checkmark$ The OB database: The OQS retrieves the full list of OBs available for observations directly from the OB database.

$\checkmark$ The OCS Sequencer: The Sequencer provides real-time information about the remaining time for the current OB being executed, which is used by the OQS to continuously recalculate the next time slot. On request from the OQS, the Sequencer translates the required instrument configuration and observing sequence defined in the OB into commands that set up the spectrograph, point the telescope, configure the fibres, rotate the tumbler, autoguide, take calibrations, take science exposures, etc.

In addition, the OQS software will need to know about any temporary (or long-term) limitations on telescope or instrument configuration, due to technical failures e.g. mIFU mode disabled, or elevation limit of telescope changed due to a problem with the dome shutters.

Detailed information about the OCS and its interactions with subsystems can be found in 10 .

\section{APPENDIX B. SKY BRIGHTNESS}

The observing condition which most strongly affects the signal-to-noise ratio of an observation is the surface brightness of the sky. The sky brightness above the observatory on La Palma can usefully be classified as dark (V-band surface brightness $\left.\sim 21.5 \mathrm{mag} \mathrm{arcsec}^{-2}\right)$, grey $\left(\sim 19.8 \mathrm{mag} \mathrm{arcsec}^{-2}\right)$ or bright $\left(\sim 18.5 \mathrm{mag} \mathrm{arcsec}^{-2}\right)$.

The sky is dark (as defined above) only in the absence of scattered moonlight, i.e. when the moon is below the horizon. The dark-of-moon sky brightness is dominated by terrestrial airglow and zodiacal light. It varies by a few tenths of a mag depending on ecliptic latitude and phase of the solar cycle as described in La Palma Night-Sky Brightness technical note $115^{\ddagger}$.

When the moon is above the horizon, the sky can be up to $\sim 4$ mag brighter than dark-of-moon, depending on lunar phase, elevation and angular distance from the target, as illustrated in Figs 6 and 7, based on the equations of Krisciunas and Schaefer (1991), ${ }_{11}^{11}$ with $V_{z e n}=21.5 \mathrm{mag} \operatorname{arcsec}^{-2}$, the mean zenith dark-sky value at the ORM and $\kappa=0.13$ mag airmass $^{-1}$, the median V-band extinction at the ORM. The moonlit sky brightness above the observatory on La Palma can probably be predicted with accuracy $\sim 20 \%$.

\footnotetext{
${ }^{\ddagger}$ http://www.ing.iac.es/Astronomy/observing/conditions/skybr/skybr.html.
} 


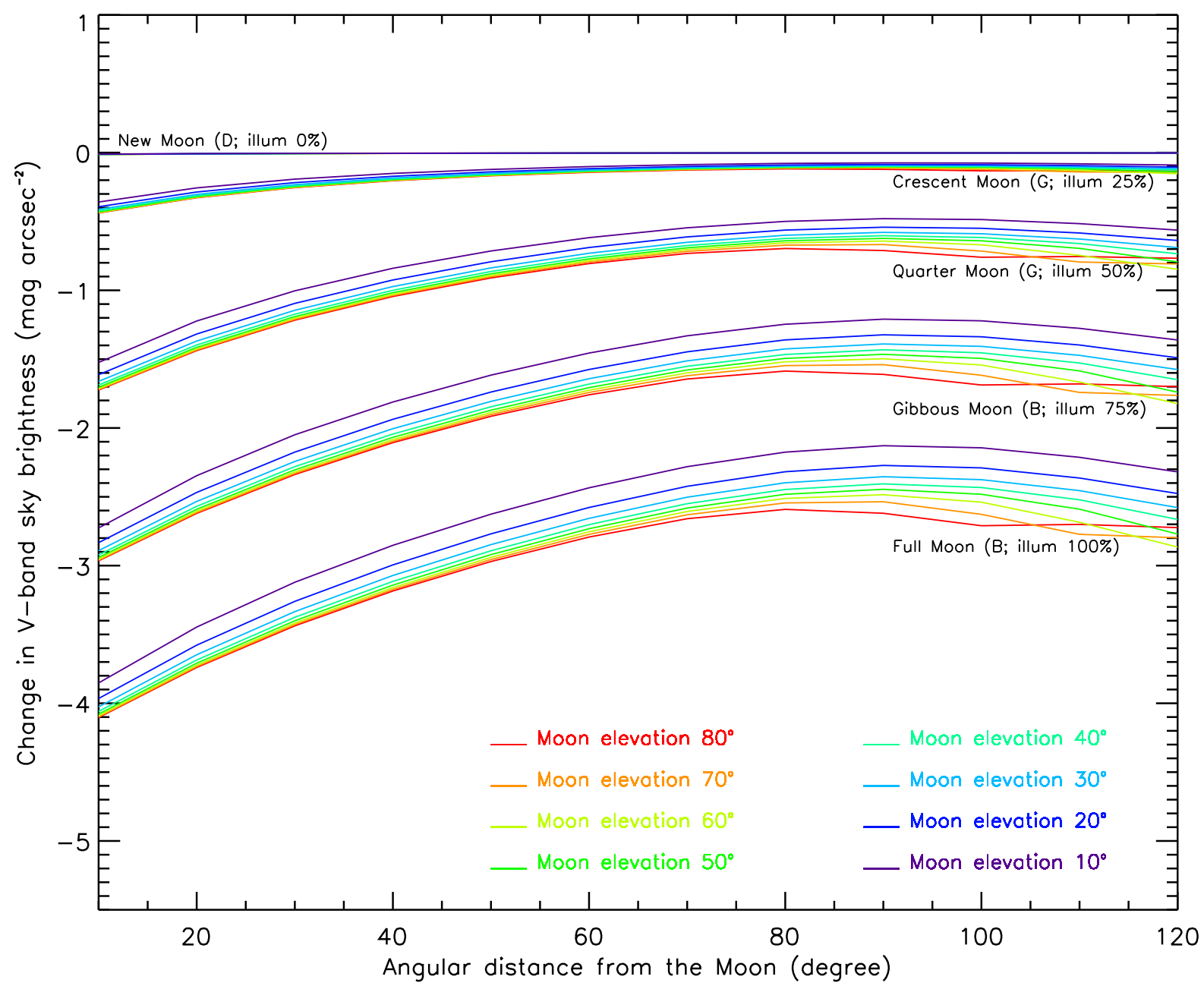

Figure 6. Change in V-band sky brightness (from dark of moon) with angular distance from the moon, for different lunar elevations (different coloured lines on each plot) and different lunar phases (as labelled). 


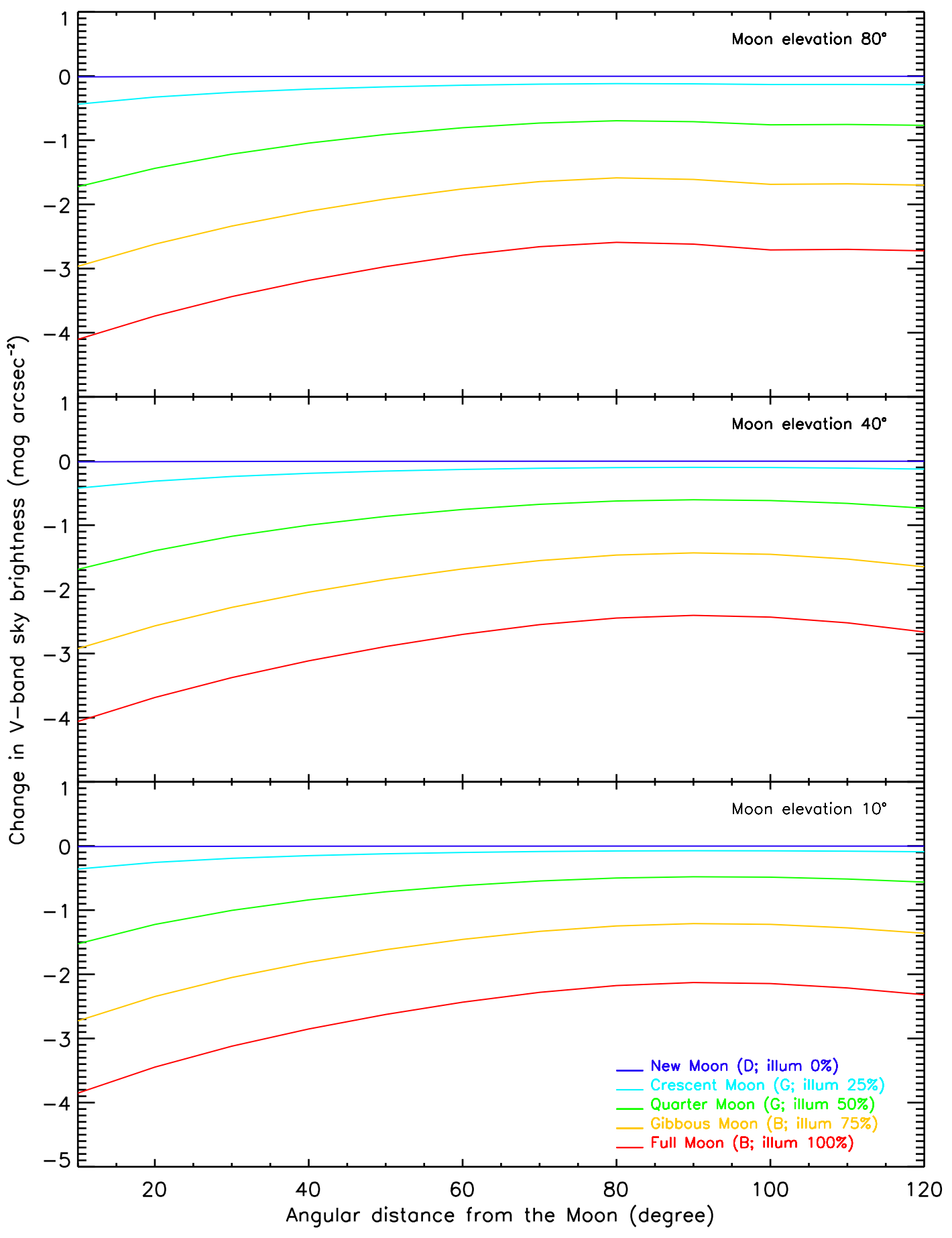

Figure 7. Change in V-band sky brightness (from dark-of-moon) with angular distance from the moon, for different moon phase in the same plot (coloured curves, as labelled) at moon elevations of 80, 40, and 10 deg (from top to bottom panel). 


\section{REFERENCES}

[1] Agócs, T., Abrams, D. C., Lhomé, E., Dee, K., Dalton, G., Middleton, K., Bonifacio, P., Aguerri, J. A. L., and Trager, S. C., "Final optical design for the WEAVE two-degree prime focus corrector," in [Ground-based and Airborne Instrumentation for Astronomy V], 9147, 914773 (July 2014).

[2] Lhomé, E., Agócs, T., Abrams, D. C., Dee, K. M., Middleton, K. F., Tosh, I. A., Jaskó, A., Connor, P., Cochrane, D., Gers, L., Jonas, G., Rakich, A., Benn, C. R., Balcells, M., Trager, S. C., Dalton, G. B., Carrasco, E., Vallenari, A., Bonifacio, P., and Aguerri, J. A. L., "Manufacturing process for the WEAVE prime focus corrector optics for the 4.2m William Hershel Telescope," in [Advances in Optical and Mechanical Technologies for Telescopes and Instrumentation II], 9912, 991245 (July 2016).

[3] Lewis, I. J., Dalton, G. B., Brock, M., Gilbert, J., Abrams, D. C., Aguerri, J. A. L., Bonifacio, P., Middleton, K., and Trager, S. C., "Fibre positioning concept for the WEAVE spectrograph at the WHT," in [Groundbased and Airborne Instrumentation for Astronomy V], 9147, 914734 (July 2014).

[4] Rogers, K., Stuik, R., Steele, I. A., Pragt, J., Middleton, K. F., Bates, S., Kragt, J., Tromp, N., Lesman, D., Lhomé, E., Dalton, G., Trager, S., Navarro, R., Abrams, D. C., Tosh, I., Jasko, A., Martin, C., O'Mahony, N., Pico, S., Cano Infantes, D., Bianca, A., Delgado, J., and Rodríguez, L. F., "The design of the WEAVE spectrograph," in [Ground-based and Airborne Instrumentation for Astronomy V], 9147, 91476H (July 2014).

[5] Domínquez, L., Cano, D., O’Mahony, N., Martín, C., Picó, S., Benn, C., Trager, S., Lhomé, É., Ridings, A., Fariña, C., Abrams, D. C., Balcells, M., Irwin, M., Lewis, J., Dalton, G., Aguerri, J. A., Bonifacio, P., Vallenari, A., and Carrasco, E., "Design of the calibration unit for the WEAVE multi-object spectrograph at the WHT," in [Ground-based and Airborne Instrumentation for Astronomy VI], 9908, 99088R (Aug. 2016).

[6] Dalton, G., Trager, S. C., Abrams, D. C., and et al., "WEAVE: the next generation wide-field spectroscopy facility for the William Herschel Telescope," in [Ground-based and Airborne Instrumentation for Astronomy $I V]$, 8446, 84460P (Sept. 2012).

[7] Dalton, G., Trager, S., Abrams, D. C., and et al., "Final design and progress of WEAVE: the next generation wide-field spectroscopy facility for the William Herschel Telescope," in [Ground-based and Airborne Instrumentation for Astronomy VI], 9908, 99081G (Aug. 2016).

[8] Terrett, D. L., Lewis, I. J., Dalton, G., Abrams, D. C., Aguerri, J. A. L., Bonifacio, P., Middleton, K., and Trager, S. C., "Fibre positioning algorithms for the WEAVE spectrograph," in [Software and Cyberinfrastructure for Astronomy III], 9152, 91520P (July 2014).

[9] Fraser, S. N., Adaptive Optimal Telecope Scheduling, PhD thesis, Liverpool John Moores University (May 2012).

[10] Picó, S., Abrams, D. C., Benn, C., Domínguez-Palmero, L., Fariña, C., Delgado, J. M., Martín, C., Cano, D., Salasnich, B., Guest, S., Gribbin, F., Skvarc, J., Gafton, E., Dalton, G., Middleton, K., Trager, S., L. Aguerri, J. A., Bonifacio, P., Vallenari, A., and Carrasco, E., "The WEAVE observatory control system," in [Archive Operations, Surveys and Datasets, SPIE, Paper No. 10704-83], (2018).

[11] Krisciunas, K. and Schaefer, B. E., "A model of the brightness of moonlight," 103, 1033-1039 (Sept. 1991). 